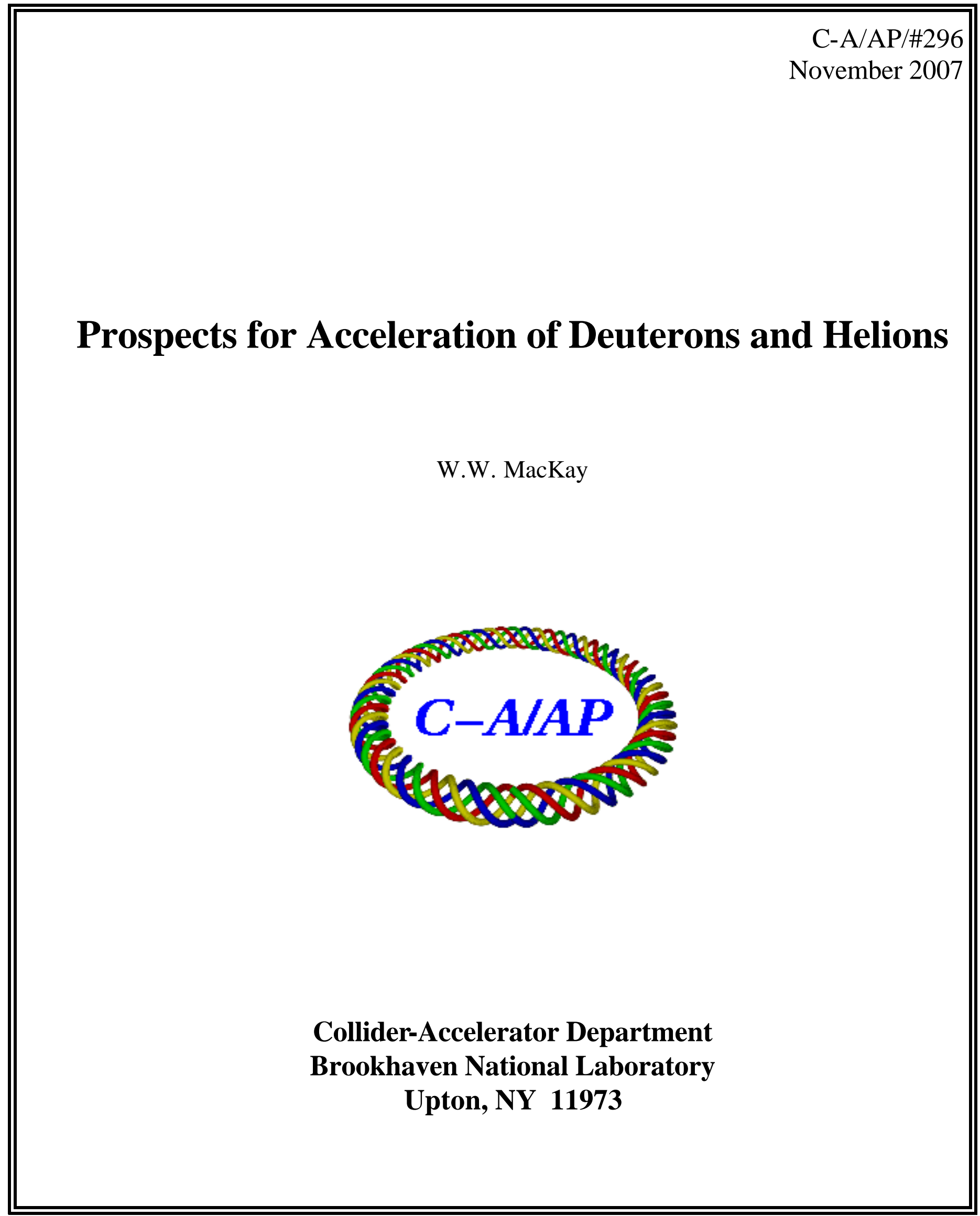




\title{
Prospects for Acceleration of Deuterons and Helions
}

\author{
W. W. MacKay \\ Brookhaven National Laboratory, Upton, NY 11973, USA.
}

\begin{abstract}
In order to study the spin structure of the neutron at high energy, a beam rich in polarized neutrons needs to be developed. The neutron has no charge and cannot be accelerated, so either deuterons or helions must be considered. When it comes to spin manipulation and stability of the polarization in a circular accelerator, it is the anomalous part of the magnetic moment which is important. If the anomaly is too small, then manipulation becomes difficult as in the case of deuterons - spin rotators and Siberian snakes become ineffective. ${ }^{3} \mathrm{He}$ nuclei appear to be the easiest choice for a polarized neutron beam. In this paper I discuss the prospects for both helions and deuterons as polarized beams in RHIC or in an electron-ion collider.
\end{abstract}

Keywords: Polarized ion beams, RHIC, deuteron, helion, proton, spin, polarization

PACS: $29.27 . \mathrm{Hj}, 29.20 . \mathrm{db}, 29.20 . \mathrm{dk}, 13.40 . \mathrm{Em}$

\section{INTRODUCTION}

The equation describing the dynamics of a relativistic particle of charge $Z e$ and mass $m$ through electromagnetic fields in an accelerator is the Lorentz force:

$$
\frac{d p^{\mu}}{d \tau}=\frac{Z e}{m} F^{\mu v} p_{v}
$$

where $p^{\mu}$ is the contravariant momentum vector, $\tau$ is the proper time, and $F^{\mu \nu}$ is the usual antisymmetric Minkowski electromagnetic field tensor. For particles with spin there will be an extremely small effect on the trajectory from the Stern-Gerlach force, but being of order $\hbar$ this is generally neglected. The precession of the spin vector is described by the relativistic torque equation (generally called the BMT or Thomas-BMT equation[1,2,3]. In covariant form the BMT equation is

$$
\frac{d S^{\mu}}{d \tau}=\frac{Z e}{m}\left[F^{\mu \nu}+\frac{g-2}{2}\left(F^{\mu \nu}+u^{\mu} F^{v \kappa} u_{\kappa}\right)\right] S_{\nu},
$$

where $u^{\mu}=p^{\mu} /(m c)$ is the proper velocity, and $S_{v}$ is the covariant spin vector[4] which is equivalent to the Pauli-Lubański vector[5] divided by mass. (The effect of the SternGerlach force on the spin is of order $\hbar^{2}$ which is even more negligible than for the trajectory.)

For a particle with $g=2$ and helicity $=1$, the spin would precess with the velocity, so that the helicity will always point in the direction of the particle's velocity, and spins initially perpendicular to the velocity would stay perpendicular to the velocity. This can provide an important check for any tracking code, but for all particles with nonzero spin 
TABLE 1. Magnetic anomalies for several isotopes

\begin{tabular}{lrrrr}
\hline & \multicolumn{1}{c}{$p$} & \multicolumn{1}{c}{${ }_{1}^{2} \mathrm{H}^{+}$} & \multicolumn{1}{c}{${ }_{1}^{3} \mathrm{H}^{+}$} & \multicolumn{1}{c}{${ }_{1}^{3} \mathrm{He}^{+2}$} \\
\hline$m\left[\mathrm{GeV} / \mathrm{c}^{2}\right]$ & 0.938272 & 1.875613 & 2.808921 & 2.808391 \\
$\mu / \mu_{\mathrm{N}}{ }^{*}$ & 2.792847 & 0.857438 & 2.972962 & -2.127498 \\
$G=(g-2) / 2$ & 1.792847 & -0.142987 & 7.918171 & -4.183963 \\
$m c^{2} / G$ & $523.3 \mathrm{MeV}$ & $-13.11 \mathrm{GeV}$ & $354.7 \mathrm{MeV}$ & $-671.2 \mathrm{MeV}$ \\
\hline
\end{tabular}

${ }^{*}$ See Ref.[7]. For the neutron $\mu_{n} / \mu_{\mathrm{N}}=-1.913043$.

used in accelerators there is always some nonzero magnetic anomaly, so helicity is not generally conserved in a circular accelerator.

The definition of the gyromagnetic ratio which I use in the BMT equation is

$$
g=\left(\frac{\mu}{\mu_{\mathrm{N}}}\right) \times \mu_{\mathrm{N}} \times \frac{2 m}{Z e \hbar I}
$$

where $\mu$ is the particle's magnetic moment, $I$ is the nuclear spin quantum number, $Z$ is the number of protons in the nucleus, and $\mu_{\mathrm{N}}=31.524512 \mathrm{eV} / \mathrm{T}$ is nuclear magneton[6]. Table 1 lists the mass, ratio of magnetic moment to nuclear magneton, $G=(g-2) / 2$, and the change in energy required for a one-unit change of $G \gamma$ for protons, deuterons, tritons, and helions. Since the deuteron has spin-1, its magnetic moment to first order is the sum of the proton and neutron moments. The moments of the triton and helion are about the same as for the unlike nucleon since the same type nucleons have moments which are paired in opposite directions and cancel to first order: $\mu_{3} \mathrm{He} \sim \mu_{n}$ and $\mu_{3} \mathrm{H} \sim \mu_{p}$.

For accelerator calculations, it is convenient to rewrite Eq. 2 in the form

$$
\frac{d \vec{S}^{*}}{d t}-\frac{e}{\gamma m}\left[(1+G \gamma) B_{\perp}+(1+G) B_{\|}+\left(G \gamma+\frac{\gamma}{1+\gamma}\right) \frac{\vec{E} \times \vec{v}}{c^{2}}\right] \times \vec{S}^{*}
$$

where the space and time coordinates $(c t, x, y, z)$ and fields $\vec{B}$ and $\vec{E}$ and velocity ${ }^{1} \vec{v}$ are given in the laboratory system, and $\vec{S}^{*}$ is referred to the instantaneous inertial rest system of the particle. (One must be very careful to realize that the $\perp$ and $\|$ subscripts on $\vec{B}$ indicate components perpendicular and parallel to the particle's actual velocity in the lab and not to the usual trajectory of the closed-orbit reference particle.) By using the spin in the rest frame, the length of the spin 3-vector is conserved, and Eq. 4 yields simple rotations of the expectation values of the nonrelativistic spin operators. In fact most algorithms for tracking the spin in accelerators ignore the tensor part of spin-1 particles such as the deuteron. In order to study the spin structure of the neutron by using polarized deuterons, we are interested primarily in deuteron beams with maximal vector polarization. Huang et al.[8] have written equations for the evolution of the spin-1 tensor

\footnotetext{
${ }^{1}$ Due to the unfortunate choice of fonts selected by the publisher, the italic letter vee $(v)$ looks almost identical to the Greek letter nu $(v)$ which is generally used in the literature for the spin tune. To reduce confusion, I write the spin tune as $v_{\mathrm{sp}}$ in this paper.
} 
TABLE 2. Approximate transfer energies for deuterons

\begin{tabular}{lrrrr}
\hline & $E / \mathbf{n}[\mathbf{G e V}]$ & $\gamma$ & $G \gamma$ & $p / q[\mathrm{Tm}]$ \\
\hline Booster inj $^{*}$ & 0.958 & 1.021 & -0.1460 & 1.291 \\
AGS inj & 1.430 & 1.525 & -0.2180 & 7.200 \\
RHIC inj & 12.134 & 12.938 & -1.8500 & 80.704 \\
RHIC max & 125.002 & 133.293 & -19.0591 & 833.904 \\
\hline The booster injection energy is a rough estimate, but it will be \\
considerably lower than for protons since the 200 MeV linac can only \\
accelerate protons.
\end{tabular}

components; however in principle, for a distribution of particles it would be simpler to track the individual spinors and then construct the average spin density matrix rather than defining tensor components for individual particles.

\section{DEUTERONS}

The problem with deuterons is that the magnetic anomaly is too small for snakes and rotators to be very effective. For example a RHIC style helical snake for protons has a central field of about $4 \mathrm{~T}$. At top energy in RHIC, a full snake for deuterons would require a peak field of about $100 \mathrm{~T}$ in the inner helices. Even if such magnets could be built, the beam at injection energy would be reflected at the entrance of the magnet rather than pass through it. The strength ${ }^{2}$ of a helical snake scales approximately with $B^{2}$. Scaling back down to $4 \mathrm{~T}$ gives an estimated strength of $\left(\frac{4}{100}\right)^{2} \sim 0.16 \%$; more detailed tracking gives the even smaller value of $0.06 \%$. While this is an extremely small value, it might be just enough to overcome the depolarizing resonances in RHIC, since the resonance strengths must also be considerably weaker than for protons.

Table 2 shows a possible scenario of transfer energies of deuterons for the RHIC complex (Fig. 1) . The single imperfection resonance (when $G \gamma$ is an integer) in the AGS might be compensated with orbit correction. It may very well be that the remaining 18 imperfection resonances in RHIC still might be compensated by a single helical snake with a partial strength of $0.06 \%$.

The RHIC spin rotators to rotate the spin from vertical to longitudinal also scale in a similar way to the snakes and will not work for deuterons. However a figure-8 style ring as proposed by Derbenev[9] could be used to obtain longitudinal and radial polarized deuterons at the interaction point. The natural spin tune of a figure- 8 ring is zero so there would be no stable spin direction. However, as Derbenev notes with a weak partial snake the spin tune is shifted slightly away from the imperfection resonance. For a fixed energy, the stable polarization direction gets locked to a specific direction at each location in the ring. Fig. 2 shows an example of such a ring with two partial snakes and $3 \pi$ worth of

\footnotetext{
2 A snake is an insertion device which rotates the spin about an axis in the horizontal plane. A full snake rotates the spin by $180^{\circ}$ in a single pass, and the strength of a partial snake is defined as the rotation angle divided by $180^{\circ}$.
} 


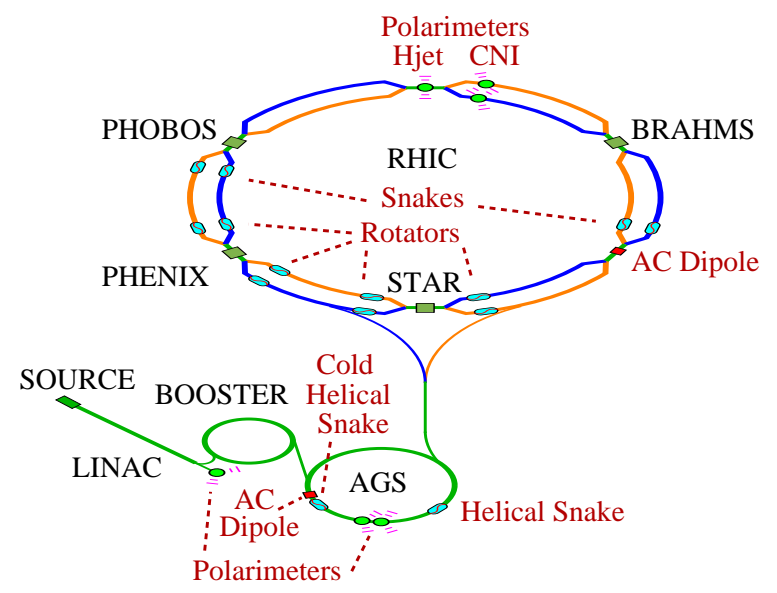

FIGURE 1. Layout of the RHIC accelerator complex for polarized protons. A different source and new lower energy ion linac would be used for polarized deuterons and helions, than the present $200 \mathrm{MeV}$ proton linac.

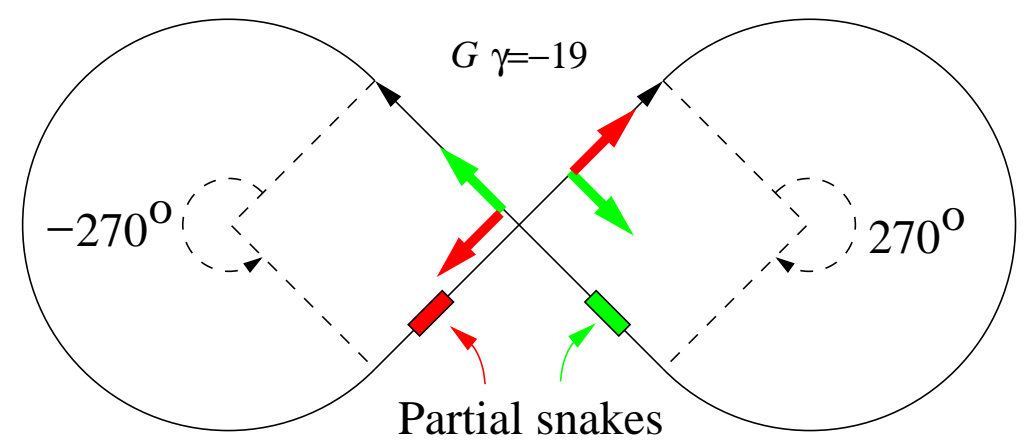

FIGURE 2. Figure-8 ring with a weak partial snakes and a spin tune close to zero. This type of ring could in principle provide longitudinally polarized deuterons at specific energies.

bending in the two arcs. If a detector were placed in one of the straight sections, then one of the two partial snakes shown would make the stable spin direction longitudinal at the detector. If the other snake were used instead, then the spin direction would be radial. Two such rings could be built to make a collider.

\section{HELIONS}

Unlike deuterons, ${ }^{3} \mathrm{He}$ ions have a much larger anomaly than even protons, so snakes and rotators can be operated at lower fields than for protons. The disadvantage with helions is that there are more depolarizing resonances which stronger and more closely spaced than for protons. Table 3 shows a possible scenario for injection and extraction energies in the Booster, AGS, and RHIC as well as the top energy for RHIC.

There are three imperfection resonances which must be crossed in the booster. Careful tuning of harmonic correctors may help reduce these resonances, or perhaps an ac dipole 
TABLE 3. Approximate transfer energies for ${ }^{3} \mathrm{He}$

\begin{tabular}{lrrrr}
\hline & $E / \mathbf{n}[\mathbf{G e V}]$ & \multicolumn{1}{c}{$\gamma$} & \multicolumn{1}{c}{$G \gamma$} & $p / q[\mathrm{Tm}]$ \\
\hline Booster inj & 1.030 & 1.100 & -4.6036 & 2.150 \\
AGS inj & 1.678 & 1.793 & -7.5000 & 6.968 \\
RHIC inj & 11.075 & 11.831 & -49.5000 & 55.216 \\
RHIC max & 166.668 & 178.039 & -744.910 & 833.904 \\
\hline
\end{tabular}
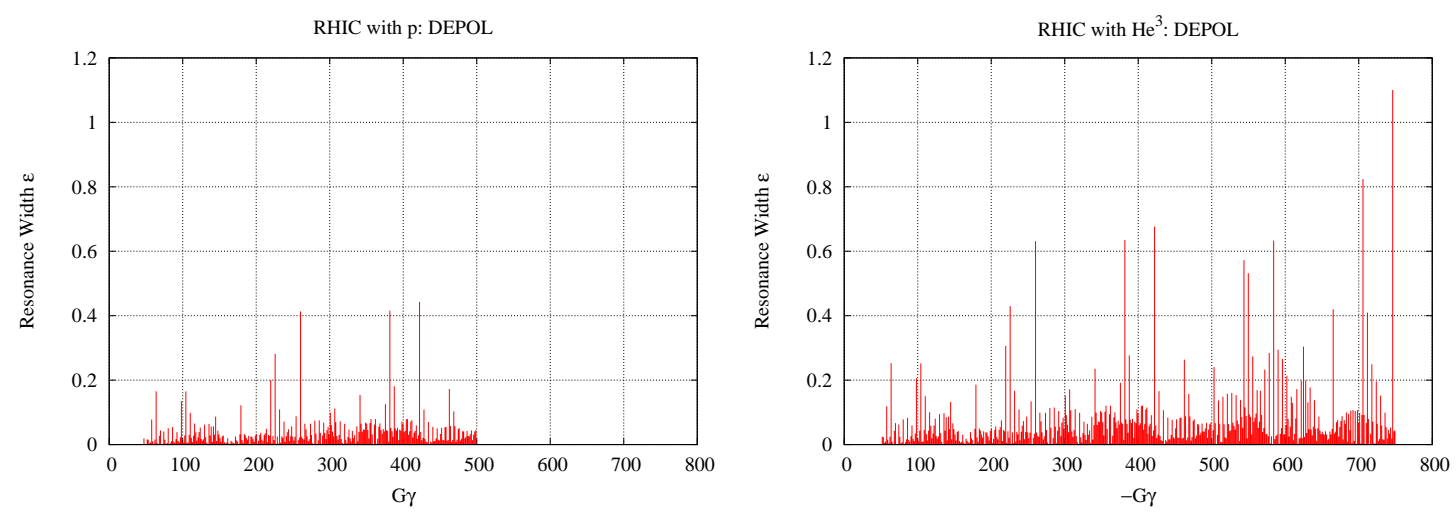

FIGURE 3. Intrinsic resonance strengths as calculated by DEPOL[10] for particles at a vertical amplitude corresponding an normalized $95 \%$ emittance of $10 \pi \times 10^{-6} \mathrm{~m}$. The left plot shows the resonance strengths for protons with a maximum value of $G \gamma_{\max }=477$ at maximum field. The right plot shows the resonance strengths for ${ }^{3} \mathrm{He}$ with $G \gamma_{\max }=743$ at maximum field. Note that DEPOL calculates these strengths without snakes.

could be employed to enhance these resonances causing a full spin flip as the beam is accelerated through each resonance.

The AGS is constructed of 12 identical sectors each containing 20 main dipoles with identically placed tune quadrupole, sextipole, and corrector magnets in each sector. The addition of two partial Siberian snakes does somewhat break this 12-fold superperiodicity; however the stronger depolarizing intrinsic resonances still tend to occur near the conditions $-G \gamma=N \times 12 \pm Q_{v}$ where $Q_{v}$ is the vertical betatron tune and $N$ is some integer. In particular with the vertical tune close to 9 , and $N=5$ we find from calculations that $|G \gamma|=51$ should be avoided; in other words, it would be better to extract the beam at a lower energy.

Fig. 3 shows depolarizing intrinsic resonance strengths as calculated by the DEPOL program[10] for both protons (left) and ${ }^{3} \mathrm{He}$ (right) in RHIC. Note that these strengths are calculated for RHIC without any Siberian snakes, so in that case the spin tune would be simply $v_{\mathrm{sp}}=G \gamma$. Since the magnitude of the anomaly is about 2.3 times larger for ${ }^{3} \mathrm{He}$ than for protons we see that there are many more lines with closer spacing and larger strengths for ${ }^{3} \mathrm{He}$. Preserving polarization of helions to top energy in RHIC will require more attention to orbit and tune control during the energy ramp, but with two full snakes good polarization should be achievable.

Since RHIC is about $1.73 \mathrm{~m}$ lower than AGS, there are vertical bends in the transport line from AGS to RHIC (see Fig. 4). There are horizontal bends between these vertical 


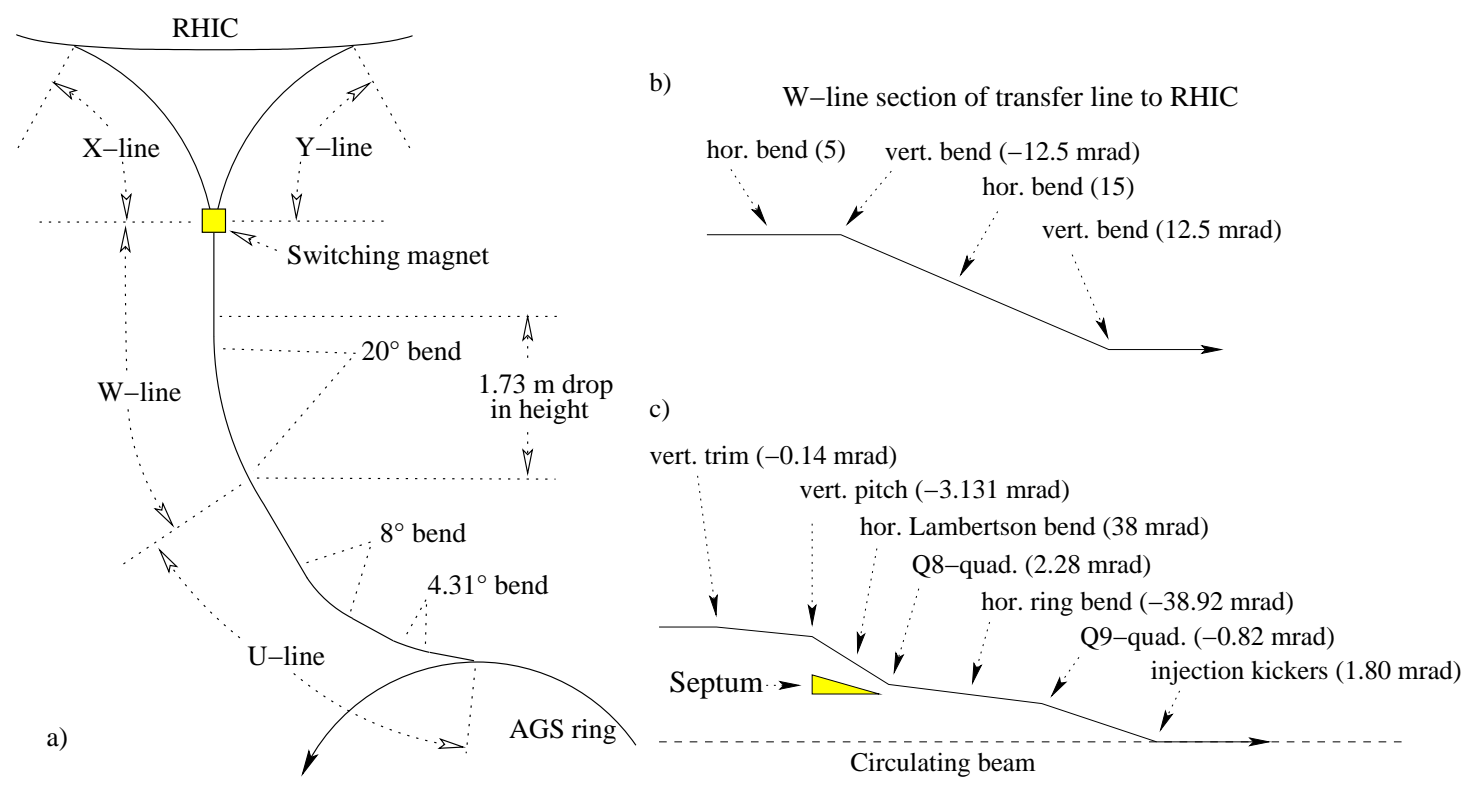

FIGURE 4. a) Horizontal layout of the transfer line from the AGS to RHIC. b) Vertical bends are interspersed between horizontal bends to drop the beam height by $1.73 \mathrm{~m}$ from AGS to RHIC. c) More mixing of vertical and horizontal bends occurs at the ends of the X-and Y-lines where the beam is injected into the RHIC rings.
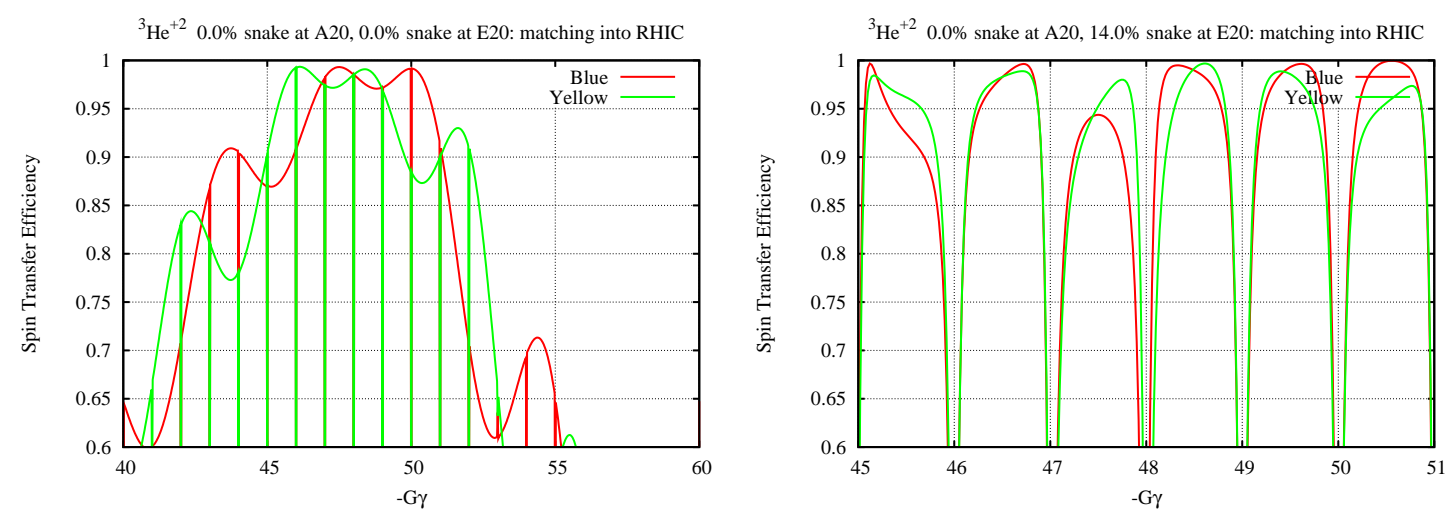

FIGURE 5. Spin matching efficiency for ${ }^{3} \mathrm{He}$ from AGS to RHIC vs $G \gamma$. Left) with no partial snakes in the AGS. Right) Spin transparency with a 14\% partial snake at the E20 straight section in the AGS. This shows a good transfer efficiency for polarization at $G \gamma=-49.5$.

bends, so the transport line is not transparent for spin at all energies. For deuterons with a very small anomalous moment, the effect of the vertical bends is negligible, but for protons $[11,12]$ and even more so for helions, it can cause a substantial loss of polarization at some energies.

The left plot of Fig. 5 shows the spin transport efficiency vs $-G \gamma$ for helions in the AGS to RHIC transport line when the partial snakes in AGS are turned off. The differences in the matching to the RHIC Blue and Yellow rings is due to the fact that the tipping of the spin away from the vertical at the entry of the switching magnet 


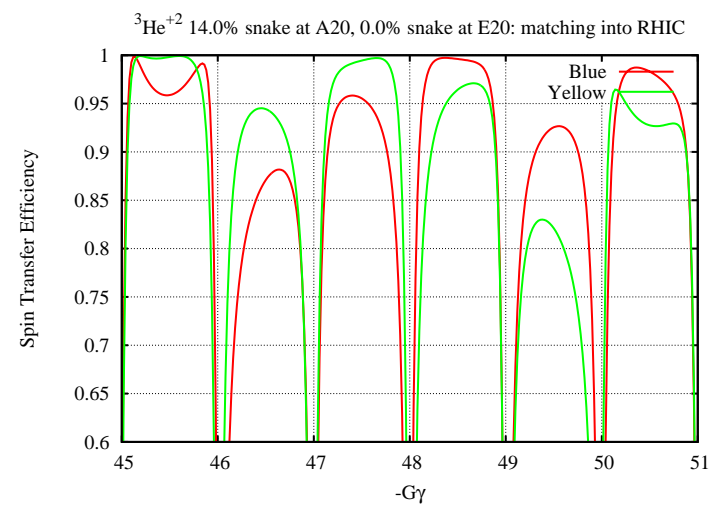

FIGURE 6. Spin matching efficiency for ${ }^{3} \mathrm{He}$ with a $14 \%$ snake at the A20 straight section in the AGS. The cold snake (superconducting) location at A20 is not as good as the warm snake (normal conducting) location at E20.(Fig. 5)

(Fig. 4a) is not purely longitudinal but has a radial component. The right plot shows how the efficiency changes when the warm snake in the E20 straight section of the AGS is turned on. The spin matching is actually improved $(>98 \%)$ by the extra tipping of the spin away from the vertical in the AGS at $-G \gamma$ of 48.5 and 49.5. To have good matching through the transport line, we would probably inject with $G \gamma=49.5$. Fig. 6 shows that the spin matching to RHIC is not quite so good for the snake at A20 as it is for the E20 snake.

\section{SUMMARY}

Longitudinally polarized deuterons do not look too promising for RHIC, since the anomalous magnetic moment is close to zero. While it may be possible to preserve vertical polarization during acceleration if the intrinsic resonance strengths can be kept smaller than the effective strength of a single partial RHIC snake for deuterons, rotation of the polarization into the longitudinal is out of the question. More study will be needed to evaluate the depolarizing resonance strengths in RHIC. It should be noted that a pair of figure- 8 rings with weak partial snakes might be able to provide longitudinally polarized deuteron collisions.

On the other hand, polarized ${ }^{3} \mathrm{He}$ should be achievable in RHIC up to $167 \mathrm{GeV} /$ nucleon since the anomalous magnetic moment is larger than for protons. The lower rigidity for ${ }^{3} \mathrm{He}$ injection into RHIC should not be a problem, since we have injected gold ions at an even lower rigidity[13]. Due to the larger anomaly, the snakes and rotators may be operated at lower currents than for protons; however the AGS warm snake which operates at a fixed current may be too strong and might need to be turned off for ${ }^{3} \mathrm{He}$ running. The cold snake at A20 which is tunable can be run at lower strengths, but its location is less optimal for spin matching to RHIC and might lower the final polarization by a few percent. The matching through the AGS to RHIC transport line could be improved with the addition of a partial solenoidal snake near the beginning of the W-line (Fig. 4). 
Polarimeters for ${ }^{3} \mathrm{He}$ (and possibly ${ }^{2} \mathrm{H}$ ) will need to be developed for RHIC and the injectors. The STAR and PHENIX experiments will also have to develop local polarimeters to ensure that there is no transverse polarization component when the spin rotators are adjusted for longitudinal polarization at the collision points.

\section{ACKNOWLEDGMENTS}

In particular I had several very enlightening discussions of spin dynamics in accelerators with Sateesh Mane. I would also like to thank S. J. Brodsky, G. Bunce, E. D. Courant, H. Huang, A. U. Luccio, L. McLarren, E. Stephenson, S. Tepikian, T. Roser A. Zelenski, for fruitful discussions on various aspects of spin dynamics of different ions species, and to M. Bai for the calculations in Fig. 3.

\section{REFERENCES}

1. L. H. Thomas, Phil. Mag. S. 7. 3, 1 (1927).

2. J. Frenkel, Zeit. Phys. 37, 243 (1926).

3. V. Bargmann, L. Michel, and V. L. Telegdi, Phys. Rev. Lett 2, 435 (1959).

4. S. Weinberg, Gravitation and Cosmology, John Wiley and Sons, New York, NY, 1972.

5. J. K. Lubański, Physica IX, 310 (1942).

6. CODATA internationally recommended values of the fundamental physical constants, NIST (2006), URL http: / / physics.nist.gov/cuu/Constants/index.html.

7. $\quad$ N. J. Stone, Atomic Data and Nuclear Data Tables 90, 75 (2005).

8. H. Huang, et al., Proc. of PAC 1993 p. 432 (1993).

9. Y. Derbenev, Proc. of EPAC 2002 p. 314 (2002).

10. E. D. Courant, and R. D. Ruth, The acceleration of polarized protons in circular accelerators, Tech. Rep. BNL-51270, BNL (1980).

11. W. W. MacKay, and N. Tsoupas, AIP Conf. Proc. 667 p. 84 (2003).

12. W. W. MacKay, et al., Proc. of EPAC 2006 p. 1795 (2006).

13. T. Satogata, Proc. of PACO7 p. 1877 (2007). 